\title{
TAIGA: results and perspectives
}

L. Kuzmichev ${ }^{1, *}$, I. Astapov ${ }^{9}$, P. Bezyazeekov ${ }^{2}$, A. Borodin ${ }^{10}$, M. Brückner ${ }^{8}$,

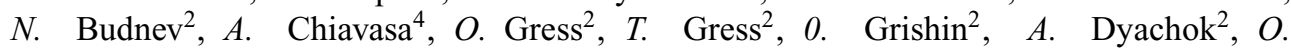
Fedorov ${ }^{2}, A$. Gafarov ${ }^{2}, A$. Garmash ${ }^{11}, V$. Grebenyuk ${ }^{10,15}, A$. Grinyuk ${ }^{10}, D$. Horns ${ }^{6}, N$. Kalmykov ${ }^{1}, Y$. Kazarina ${ }^{2}, V$. Kindin $^{9}, V$. Kiryuhin $^{2}, R$. Kokoulin ${ }^{9}, K$. Kompaniets ${ }^{9}, E$. Korosteleva $^{1}$, $V$. Kozhin ${ }^{1}$, E. Kravchenko ${ }^{11,12}$, A. Kryukov ${ }^{1}, A$. Lagutin ${ }^{14}$, $V$. Lenok ${ }^{2}$, $B$. Lubsandorzhiev ${ }^{3}, N$. Lubsandorzhiev ${ }^{1}, R$. Mirgazov $^{2}, R$. Mirzoyan $^{5,2}, R$. Monkhoev ${ }^{2}, E$. Osipova ${ }^{1}, A$. Pakharukov ${ }^{2}, A$. Pan $^{10}, M$. Panasyuk $^{1}, L$. Pankov $^{2}, A$. Petrukhin $^{9}, V$. Poleschuk $^{2}$,

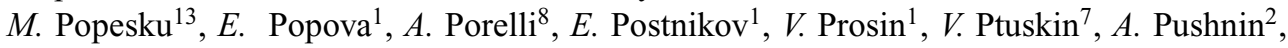
R. Raikin ${ }^{14}, G$. Rubtsov ${ }^{3}, E . \mathrm{Rybov}^{2}, Y$. Sagan ${ }^{10,15}, V$. Samoliga $^{1}, Y u$. Semeney ${ }^{2}, A$. Silaev $^{1}, A$. Silaev(junior) ${ }^{1}, A$. Sidorenkov ${ }^{3}, A$. Skurikhin $^{1}, M$. Slunecka ${ }^{10}, A$. Sokolov ${ }^{11,12}$, C. Spiering ${ }^{8}, L$. Sveshnikova ${ }^{1}, V$. Tabolenko ${ }^{2}, B$. Tarashansky ${ }^{2}, L$. Tkachev ${ }^{10,15}, M$. Tluczykont $^{6}, N$. Ushakov ${ }^{3}, A$. Vaidyanathan ${ }^{12}, P$. Volchugov ${ }^{1}, D$. Voronin $^{3}, R$. Wischnewski ${ }^{8}$, $A$. Zagorodnikov ${ }^{2}, V$. Zurbanov ${ }^{2}, D$. Zhurov $^{2}$, and $I$. Yashin ${ }^{9}$

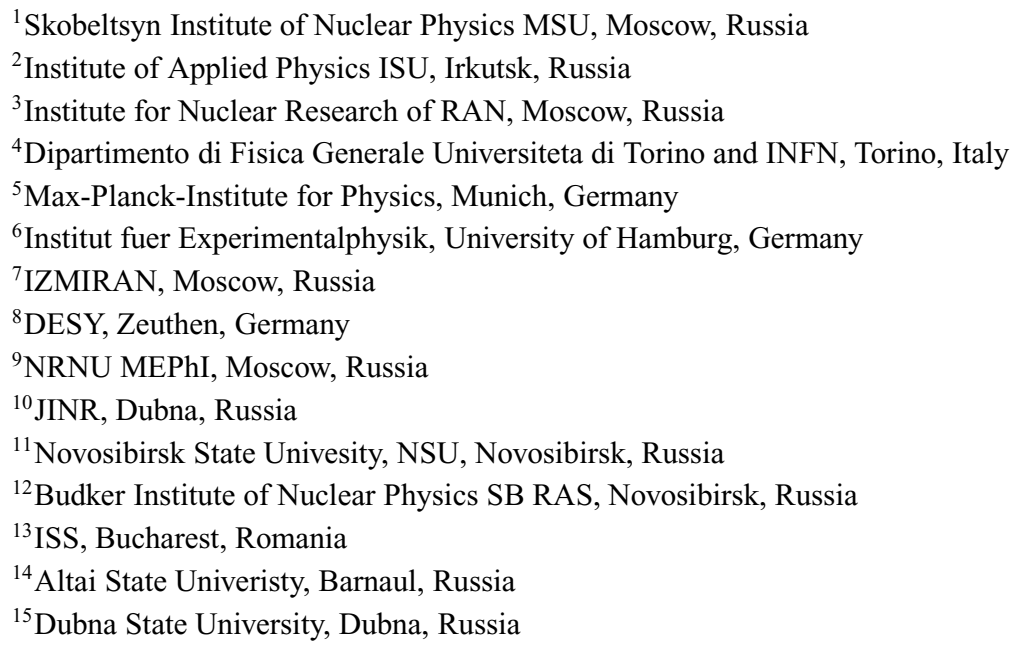

Abstract. In this talk, we describe the status and the perspectives of the hybrid Air Shower Array TAIGA (Tunka Advanced Instrument for cosmic rays and Gamma Astronomy) which is currently under construction in the Tunka Valley close to Lake Baikal and is taking data in its initial configurations. TAIGA is designed for the study of gamma rays and charged cosmic rays in the energy range of $10^{13} \mathrm{eV}-10^{18} \mathrm{eV}$. It has the potential to play an important role in the search for Galactic Pevatrons and within a multi-messenger approach to explore the high-energy sky.

\footnotetext{
*e-mail: kuz@dec1.sinp.msu.ru
} 


\section{Introduction}

The first of the presently operating installations in the Tunka valley was the Cherenkov light array Tunka-133, with $\sim 3 \mathrm{~km}^{2}$ geometrical area, taking data since 2009 . Tunka-133 is devoted to the study of energy spectrum and mass composition of cosmic rays in the energy range from $6 \times 10^{15}$ to $10^{18} \mathrm{eV}$. At present, three arrays are operated to study charged cosmic rays: Tunka-133 [1], the radio antennas Tunka-REX [3] and the scintillation array TunkaGrande [4]. Most of the ongoing effort, however, is focused on the construction of the first stage of TAIGA (Tunka Advanced Instrument for cosmic ray physics and Gamma Astronomy). This array is designed to study gamma rays and charged cosmic rays in the energy range of $10^{13}-10^{18} \mathrm{eV}$. In the following we will solely focus on the TAIGA detector.

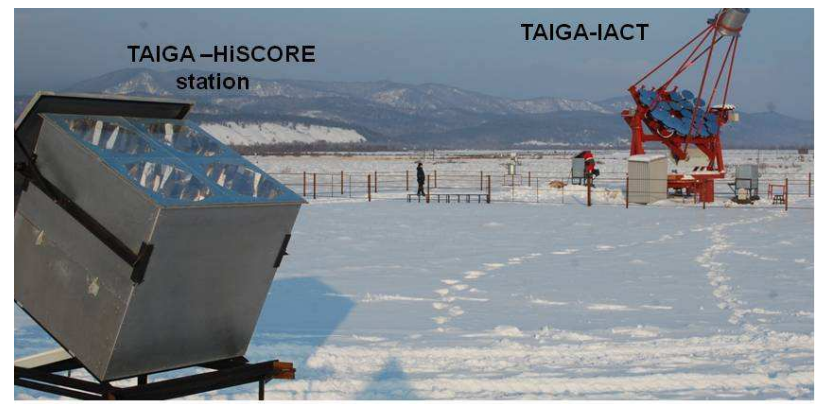

Figure 1. TAIGA-HiSCORE station and TAIGA-IACT

TAIGA will include a network of wide field of view (0.6 sr) timing Cherenkov light stations, named TAIGA-HiSCORE [5],[6] (High Sensitivity Cosmic Origin Explorer), and up to 16 imaging atmospheric Cherenkov telescopes (Fig. 1), covering an area of $10 \mathrm{~km}^{2}$. The capabilities of these Cherenkov arrays are enhanced by muon detectors (TAIGA-Muon) with a total coverage of $2000 \mathrm{~m}^{2}$, distributed over an area of $1 \mathrm{~km}^{2}$. The advantage of a few IACTs added to the wide-angle timing array is their better gamma/hadron separation by image parameter information, while core position, direction and energy can be better reconstructed by the timing array. Combination of information from both components gives a superior result compared to the performance of each single component. The detection sensitivity of a $10 \mathrm{~km}^{2}$ installation for poinr sources in the energy range of $30-200 \mathrm{TeV}$ is expected to be $5 \cdot 10^{-14} \mathrm{TeV} \mathrm{cm} \mathrm{sec}^{-1}$ for $500 \mathrm{~h}$ of observation or 10 detected events.

\section{TAIGA-HiSCORE}

As of August 2018, the prototype of TAIGA consists of 54 TAIGA-HiSCORE stations and one IACT. The 54 optical stations are distributed in a regular grid over a surface area of 0.5 $\mathrm{km}^{2}$ with an inter-station spacing of $106 \mathrm{~m}$. All stations are tilted into the southern direction by $25^{\circ}$ to increase the time for the study gamma-quanta fluxes from the first test object - the Crab Nebula. Each optical station contains four large area PMTs with 20 or $25 \mathrm{~cm}$ diameter, EMI ET9352KB and Hamamatsu R5912 or R7081. Each PMT has a Winston cone with $0.4 \mathrm{~m}$ diameter and a $30^{\circ}$ viewing angle (field of view $\sim 0.6 \mathrm{sr}$ ). Each station is connected with the DAQ center by a fiber optic cable for data transfer and synchronization stability of the stations reaches $\sim 0.2 \mathrm{~ns}$ and is maintained by two independent systems (custom $100 \mathrm{MHz}$ system and White-Rabbit)[7],[8]. Precision calibration is achieved by external light sources. 


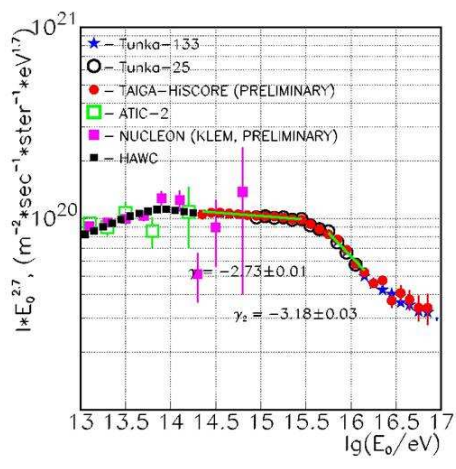

Figure 2. The energy spectrum of primary cosmic rays from the data of the TAIGA-HiSCORE array in comparison with other experiments.

Signals from anode and intermediate dynode are digitized with a step of $0.5 \mathrm{~ns}$ by a special board based on the DRS-4 chip. The reconstruction of shower parameters was performed using algorithms developed for the Tunka-133 [1, 2] and for TAIGA-HiSCORE [9]. The angular resolution is equal to $0.4^{\circ}-0.5^{\circ}$ for events with $4-5$ hit stations and about $0.1^{\circ}$ for events with more than 10 hit stations [10]. The accuracy of the reconstruction procedure was checked on MC simulation as well as with experimental data. The latter are presented in [11],[12] (chess-board method).

Approximately twice per month during the season of 2016-2107, HiSCORE recorded signals from CATS (Cloud Aerosol Transport System) - the lidar onboard the ISS [13]. The lidar operates at a wavelength of $532 \mathrm{~nm}$ with a frequency of $4 \mathrm{kHz}$. On March 23, 2017 the signal from the lidar was also registered with the MASTER optical telescope [14], located at a distance of $500 \mathrm{~m}$ from the center of the installation. Comparison of the angular position of events with maximum amplitudes in HiSCORE stations, restored with calibrated delays, and the position of the point of maximum brightness according to the MASTER telescope showed that the absolute angular accuracy of the array is not worse than $0.1^{\circ}$ [15].

Naturally, HiSCORE can also measure the spectrum of charged cosmic rays. The preliminary cosmic rays spectrum as measured with HiSCORE is compared in Fig. 2 to the results of previous experiments in the Tunka Valley, as well as with the results of the balloon experiment ATIC-2 [16], the satellite experiment NUCLEON [17] and a new experimental spectrum obtained by the HAWC experiment [18] in Mexico.

\section{TAIGA-IACT}

The IACTs have an optical design of the Davis-Cotton type with 29 mirrors, each of $60 \mathrm{~cm}$ diameter and a focal length of $4.75 \mathrm{~m}$. The imaging camera comprises 560 PMTs of XP1911 type (Photonics) of $19 \mathrm{~mm}$ diameter. The FOV of the camera is $9.6^{\circ}$ (each pixel has an aperture $0.36^{\circ}$ ), with a Point Spread Function (PSF) of $0.07^{\circ}$. The CCD-camera Prosilica GC1380 is installed at a distance of $1 \mathrm{~m}$ from the telescope optical axis on the mirror dish. The CCD-camera is used for checking the telescope pointing direction. It has $1360 \times 1024$ pixels resolution and $31.4^{\circ} \times 23.6^{\circ}$ field of view. Each axis of the telescope is equipped with a Phytron hybrid stepper motor, a 17-bit shaft encoder and end-of-zone switches connected to the PhyMOTION control unit. 


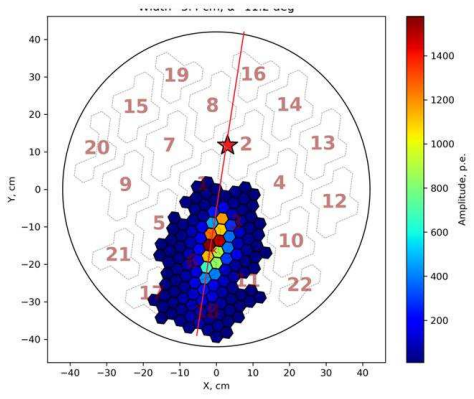

Figure 3. Example of a hadron-like joint event. Image parameters: Size $=18500$ p.e., Width $=0.4^{\circ}$, Alfa $=11^{\circ}$.

The camera consists of identical clusters, each based on 28 PMTs. The basis of the cluster electronics is a 64-channel ASIC MAROC-3. Each channel includes a preamplifier with adjustable gain, a charge sensitive amplifier and a comparator with adjustable threshold. This chip has a multiplexed analog output signal which is proportional to the input charge. The chip is connected to a 12-bit external ADC. The signal from each PMT is split and fed into 2 MAROC-3 channels with a gains difference of 30 . This results in a full dynamic range of 3000 photoelectrons.

After the installation of the shaft encoder on the axis in September 2017, the commissioning of the TAIGA-IACT telescope tracking system was started and first source observations were performed in October 2017.

In the 2017-2018 season the telescope pointing accuracy was $\sim 0.05^{\circ}$ for the corrected observations in March (mostly Mrk 421) and around $0.1^{\circ}$ for the other runs (Crab Nebula and Mrk 421)[19]. The Crab Nebula and Mrk-421 were planned to be observed for 120 hours during the last season. Unfortunately due to bad weather and a number of technical problems it was possible to observe these sources only for about 25 hours. We have obtained first promising results but have decided to present results only after 50 hours of observation and after two independent analyses.

The search for joint events recorded by the telescope and the TAIGA-HiSCORE array was carried out while tracking the Crab Nebula [20]. Fig. 3 shows an example of a joint hadron-like event detected by the telescope and TAIGA-HiSCORE. The asterisk marks the projection of the EAS core position on the plane of the telescope camera with the introduction of a scaling factor $\mathrm{R}_{p}(\mathrm{~cm}) / \mathrm{R}_{c}(\mathrm{~cm})=1500$, where $\mathrm{R}_{p}$ is the distance from telescope to the EAS core position and $\mathrm{R}_{c}$ the distance between camera center and asterisk. The line on the picture is directed to the EAS core. For events coming from the source to which the telescope is oriented, the line connecting the projection of the EAS axis and the center of gravity of the image should cross the center of the camera. The same event was detected by 15 stations of the TAIGA-HiSCORE array: $\mathrm{E}=840 \mathrm{TeV}, \theta=30.1^{\circ}, \phi=33.6^{\circ}, \mathrm{R}_{p}=134 \mathrm{~m}$, the angle between direction of the shower, reconstructed by timing array, and the telescope pointing direction is $\psi=0.47^{\circ}$.

17,000 joint events with an image size $\geq 60$ p.e. were selected. These events were detected by the first 30 stations of the TAIGA-HiSCORE array, with an area of $0.25 \mathrm{~km}^{2}$. Fig 4 A shows the Width distributions for joint events with size between 1000 and 3000 pe. 


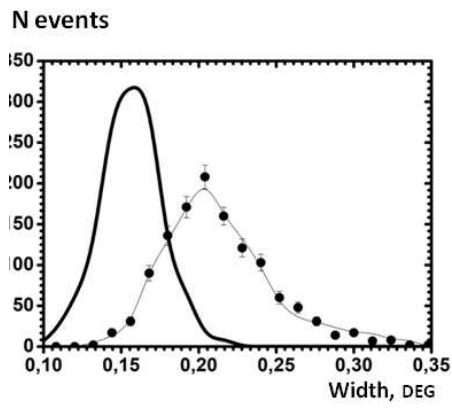

A

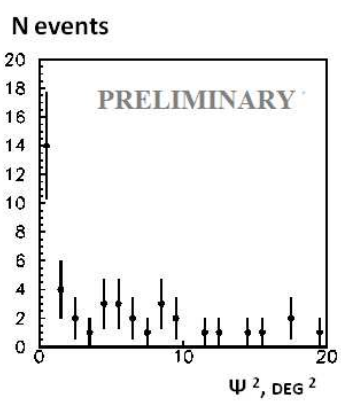

B

Figure 4. A:Image Width distribution for joint events. Solid circles - experiment. Thin solid line - MC (cosmic rays), thick solid line - MC (gamma quanta). B: $\Psi^{2}$ distribution for hybrid events after cuts (preliminary results).

The thin solid line indicates the MC simulations for a similar sample of events, obtained from experiment. The thick solid line at this figure is the Width distributions for gamma rays for the mentioned sample. Selecting $60 \%$ of gamma-events (Width $\leq 0.17^{\circ}$, Alfa $\leq 15^{\circ}$ ) we suppressed the hadron background by a factor of $\sim 100$. With such cuts selected for event sample we have a Q-factor of 5.

After applying Width $\left(\leq 0.18^{\circ}\right)$, Alfa $\left(\leq 15^{\circ}\right)$ and Dist. $\left(\leq 2.5^{\circ}\right)$ cuts, there remain 42 events with $\Psi \leq 4.5^{\circ}, \Psi$ - being the angle between direction of showers, reconstructed by TAIGA-HiSCORE and the direction of the Crab Nebula. The $\Psi^{2}$ distribution of these hybrid events after cuts is presented on Fig 4B. For 14 of these events, with energies between 45 and $60 \mathrm{TeV}, \Psi \leq 1^{\circ}$. We should mention that for such energies multiplicity of hit HiSCORE stations is only $4-5$ and the angular resolution is $0.4^{\circ}-0.5^{\circ}$. These events may be considered as candidates for first gamma-like events selected by the hybrid approach.

\section{Conclusions}

The first significant steps towards completing the gamma-ray observatory TAIGA have been performed. The first seasons of operation of TAIGA-HiSCORE and the first TAIGA-IACT demonstrated good performance of the installation and showed yet preliminary but very interesting results which will be presented after confirmation by more data and two idependent analyses. During the winter season 2018-2019, TAIGA will include 54 operational HiSCORE stations arranged over an area of $0.5 \mathrm{~km}^{2}$, and one IACT. During the next year it is planned to finish deployment of the first stage of TAIGA with 110 TAIGA-HiSCORE stations and 3 IACTs on an area of $1 \mathrm{~km}^{2}$. The expected integral sensitivity for 300 hours of source observation (about $2-3$ seasons of operation) in the range $30-200 \mathrm{TeV}$ is about $5 \cdot 10^{-13} \mathrm{TeV}$ $\mathrm{cm}^{-2} \sec ^{-1}$ (Fig 5).

Our primary goal is the identification of Galactic Pevatrons and corresponding extragalactic sources. With our unique geographical location and the wide-angle capabilities we also hope to be able to contribute to multi-messenger studies as discussed on this workshop.

\section{Acknowlegements}

This work was supported by the Russian Federation Ministry of Education and Science (projects 14.593.21.0005 (Tunka shared core facilities, unique identifica- 


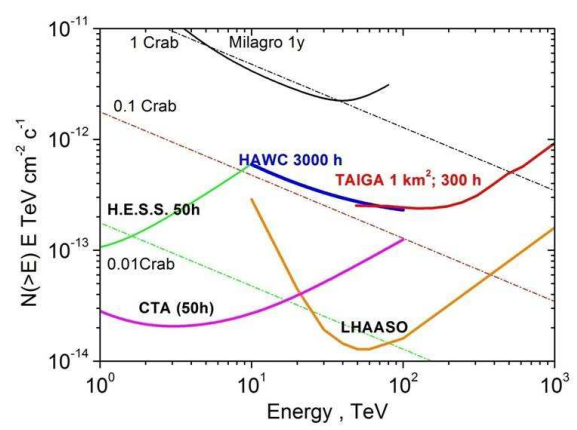

Figure 5. Integral sensitivity for detection of point sources.

tor RFMEFI59317X0005), 3.10131.2017/NM, 2017-14-595-0001-003, 3.9678.2017/8.9, 3.904.2017/4.6, 3.5917.2017/BCh, 3.6787.2017/7.8, 3.5939.2017/8.9, 1.6790.2017/7.8), the Russian Foundation for Basic Research (Grants 16-02- 00738, 16-32-00329, 17-02-00905, 16-29-13035), the German Helmholtz Association (grant HRJRG-303), the DFG (grant TL 51-3) and the European Union's Horizon 2020 program under grant agreement No. 653477.

\section{References}

[1] S. Berezhnev et al. (Tunka Collaboration), NIM A 692, 98 (2012)

[2] Prosin V.V. et al. (Tunka Collaboration), NIM A 756, 94 (2014)

[3] P.A. Bezyazeekov et al. (Tunka-REX Collaboration), JCAP 01, 052 (2016)

[4] R. Monhoev et al. (Tunka Collaboration), Bull. Russian Ac. of Sciences: Physics, 81, 504 (2017)

[5] M. Tluczykont et al., Adv.Space Res. 48,1935-1941 (2011)

[6] M. Tluczykont et al., Astropaticle Phys. 56, 42 (2014)

[7] O. Gress et al. (TAIGA Collaboration), NIM A 845, 367 (2017)

[8] N.Budnev et al.(TAIGA Collaboration),PoS ICRC2017, 768 (2018)

[9] D.Hampf et al., NIM A 712, 137 (2013)

[10] L.Kuzmichev et al. (TAIGA Collaboration), EPJ Web of Conf. 145, 01001 (2017)

[11] M.Tluczykont et al. (TAIGA Collaboration), EPJ Web of Conf. 136, 03008 (2017)

[12] A.Porelli et al. (TAIGA Collaboration) Proceeding ECRS 2014, J. of Phys:Conf.Ser. 632, 012041 (2015)

[13] http://cats.gsfc.nasa.gov/

[14] V. Lipunov et al., Adv. Astron. 2010, 349171 (2010)

[15] A.Porelli et al. (TAIGA Collaboration) Pos (ICRC2017) 754 (2017)

[16] A.Panov et al., Bull. Russ. Acad. Sci. Phys. 73 5, 564 (2011)

[17] N.Gorbunov et al. (Nucleon Collaboration), arXiv:1809.05333

[18] Alfaro et al. (HAWC Collaboration) Phys. Rev. D 96, 122001 (2017)

[19] D.Zhurov et al. (TAIGA Collaboration), Report at 26th ECRS, Barnaul, 2018, to be published in Journal of Physics: Conference Series

[20] L.Sveshnikova et al. (TAIGA Collaboration) Report at 26th ECRS, Barnaul, 2018, to be published in Bull. Russ. Acad. Sci. Phys. 2019 